\title{
ASOCIACIÓN DEL POLIMORFISMO GENÉTICO DE LA INTERLEUCINA -1BETA CON PERIODONTITIS CRÓNICA EN ADULTOS PERUANOS
}

\author{
Ivonne F. Reyes-Mandujano, 1,2,a José E. Olivera-García1,2,3,b , Manuel Taboada-Vega ${ }^{1,4, c}$, Patricia Galvan-Sanchez $^{1,2, d}$, \\ Amilcar Azcarza-Acuña ${ }^{1, e}$, Aldo M.Yactayo-Flores ${ }^{1,2, f}$, Luis A. Zuñiga-Ccoicca ${ }^{1,2, g}$, Evelyn V. Leiva-Pachas ${ }^{1,2, h}$,
} Efraín Paccori-García ${ }^{1,5, i}$

\section{RESUMEN}

Objetivos. Determinar la asociación del polimorfismo IL-1B C(+3953/4)T con la periodontitis crónica en adultos. Materiales y métodos. Estudio de casos y controles, se incluyeron personas de 18 a 64 años muestreados voluntariamente aplicando el consentimiento informado a través de campañas de salud, realizadas durante el 2012, en diferentes zonas de la ciudad de Lima con características socioeconómicas semejantes. Odontólogos especialistas en periodoncia realizaron el diagnóstico del estado periodontal de los participantes, la genotipificación se realizó a través de la técnica de PCR-RFLP. Los datos fueron analizados mediante regresión logística. Resultados. Los factores asociados con la periodontitis crónica fueron ser mayor de 46 años (OR: 7,58, IC $95 \%: 3,32-17,30$ ), tener grado de instrucción superior (OR: 0,43, IC $95 \%$ : 0,27-0,98), la presencia del alelo 2 en el polimorfismo de la IL-1B y el genotipo positivo (2-2) estuvo asociado con la presencia de periodontitis crónica (OR: 2,06, IC $95 \%$ : 1,01-4,21). Conclusiones. La presencia del alelo 2 en el polimorfismo de la IL-1B y el genotipo positivo (2-2) confiere mayor riesgo para el desarrollo de periodontitis crónica en la población de adultos peruanos que fueron estudiados.

Palabras clave: Reacción en cadena de la polimerasa; Citoquinas; Periodontitis crónica; Polimorfismo genético. (fuente: DeCS BIREME).

\section{CONNECTION BETWEEN GENETIC POLYMORPHISM OF INTERLEUKIN -1BETA WITH CHRONIC PERIODONTITIS IN PERUVIAN ADULTS}

\begin{abstract}
Objectives. To determine the connection between polymorphism IL-1B C $(+3953 / 4) T$ and chronic periodontitis in adults. Materials and Methods. Case and control study. Individuals between 18 and 64 years of age were included; they were recruited through healthcare campaigns carried out in 2012 in different areas of the city of Lima with similar socio-economic characteristics. Dentists specialized in periodontics performed the diagnosis of the periodontal state of participants; genotyping was made through the PCR-RFLP technique. The data were analyzed by logistic regression. Results. The factors associated with chronic periodontitis were: age over 46 years (OR: 7.50, Cl 95\%: 1.85-6.37), higher education level achieved (OR: 0.43, Cl 95\%: 0.27-0.98), the presence of allele 2 in the polymorphism of IL-1B. The positive genotype (2-2) was associated with the presence of chronic periodontitis (OR: 2.06, Cl 95\%: 1.01-4.21). Conclusions. The presence of allele 2 in the polymorphism of IL-1B and the positive genotype (2-2) confers greater risk for the development of chronic periodontitis in the population of Peruvian adults under study.
\end{abstract}

Keywords: Polymerase Chain Reaction; Cytokines; Chronic Periodontitis; Genetic Polymorphism. (source: MeSH NLM).

Reyes Mandujano I.F. Asociación del Polimorfismo Genético de las IL-1A C(-889)T y IL-1B C(+3953/4)T con Periodontitis Crónica en una Población Peruana [tesis de maestría]. Lima: Centro de Investigación de Bioquímica y Nutrición “Alberto Guzmán Barrón”. Universidad Nacional de San Marcos; 2015.

\footnotetext{
Centro de Investigación de Bioquímica y Nutrición “Alberto Guzmán Barrón”. Universidad Nacional de San Marcos. Lima, Perú. Facultad de Ciencias Biológicas. Universidad Nacional de San Marcos. Lima, Perú

Instituto de Investigación Estomatológica de la Facultad de Odontología. Universidad Nacional de San Marcos. Lima, Perú.

Departamento Académico de Ciencias Básicas de la Facultad de Odontología. Universidad Nacional Mayor de San Marcos, Lima, Perú.

Facultad de Odontologia. Universidad San Martín de Porras. Lima, Perú.

Biólogo con mención en biología celular y genética, magíster en biología molecular; ${ }^{\mathrm{b}}$ biólogo con mención en genética , magíster en bioquímica; ${ }^{\mathrm{c}}$ cirujano dentista, doctor en odontología; ${ }^{\mathrm{d}}$ biólogo con mención en biología celular y genética; ${ }^{\mathrm{e}}$ cirujano dentista, maestría en periodoncia; ${ }^{\mathrm{f}}$ biólogo con mención en zoología; ${ }^{\mathrm{g}}$ bachiller en genética y biotecnología; ${ }^{\mathrm{h}}$ bachiller en genética y biotecnología; ${ }^{\mathrm{i}}$ cirujano dentista, magister en docencia e investigacion en estomatologia.

Recibido: 04/07/2017 Aprobado: 31/01/2018 En línea: 05/04/2018
}

Citar como: Reyes-Mandujano IF, Olivera-García JE, Taboada-Vega M, Galvan-Sanchez P, Azcarza-Acuña A, Yactayo-Flores AM, et al. Asociación del polimorfismo genético de la interleucina -1beta con periodontitis crónica en adultos peruanos. Rev Peru Med Exp Salud Publica. 2018;35(1):9-14. doi: 10.17843/rpmesp.2018.351.3595 


\section{INTRODUCCIÓN}

La periodontitis es una enfermedad infecciosa crónica del tejido que soportan los dientes (ligamento periodontal, cemento radicular y hueso alveolar) y es considerada como la causa más común de la pérdida de dientes entre los adultos a nivel mundial (1); directamente no es una enfermedad mortal; pero, podría predisponer a varias enfermedades sistémicas serias ${ }^{(2,3)}$.

La patogenia de la periodontitis se inicia con la colonización, en el microambiente gingival de un huésped susceptible, de bacterias cariogénicas. Posteriormente, se produce la destrucción tisular característica de la periodontitis, como parte de una respuesta del huésped contra estos organismos. Este proceso tiene una estrecha relación con varios factores, entre ellos la condición genética de la persona ${ }^{(4-7)}$.

Aunque, comercialmente, existen pruebas que determinan la susceptibilidad de los pacientes a desarrollar periodontitis basados en polimorfismos de la IL-1B; la especificidad de los marcadores genéticos asociados con el incremento de la producción de la IL-1 $\beta$ sigue siendo de interés para la comunidad científica, debido a su correlación con la gravedad de la periodontitis; y a que aún no es claro el mecanismo y la influencia de factores tales como la etnicidad sobre la especificidad y sensibilidad de estas pruebas ${ }^{(8)}$; dado que estas han sido evaluados sólo en ciertos grupos poblacionales ${ }^{(9)}$.

En el Perú, se ha evaluado el gen TNF-alfa (-308G/A) ${ }^{(10)}$, pero no se ha encontrado relación con la periodontitis crónica. El polimorfismo de la IL-1B C(+3953/4)T es considerado, en algunas poblaciones, un factor de riesgo potencial para la destrucción periodontal ${ }^{(11)}$; en tal sentido, el objetivo del presente estudio fue determinar si existe relación entre el polimorfismo genético de la interleucina -1 beta en el desarrollo de la periodontitis crónica en una muestra de adultos peruanos.

\section{MATERIALES Y MÉTODOS}

\section{DISEÑO Y POBLACIÓN DE ESTUDIO}

Se realizó un estudio de casos y controles siendo considerados como casos las personas con periodontitis crónica y los controles personas periodontalmente sanos. La población se compuso de personas entre 18 a 65 años reclutadas a través de campañas de salud, realizadas durante el 2012, en diferentes zonas de la ciudad de Lima con características socioeconómicas semejantes. 190 de 269 personas reclutadas, cumplieron con los criterios de inclusión (peruanos al igual que sus progenitores; que deseen voluntariamente participar del estudio). No fueron incluidos en el estudio personas con enfermedades que favorezcan el desarrollo de la periodontitis (diabetes, hepatitis, $\mathrm{VIH}$, desordenes inmunitarios o de coagulación), se excluyeron gestantes o que esten dando de lactar, fumadores, personas que usen braquets, prótesis dental o tengan periodontitis agresiva.

\section{MENSAJES CLAVE}

Motivación para realizar el estudio. La periodontitis es una causa común en la pérdida de dientes entre los adultos. En algunas poblaciones el polimorfismo de la IL-1B C $(+3953 / 4) \mathrm{T}$ es considerado un factor de riesgo para la destrucción periodontal. Sin embargo, en población peruana no se ha encontrado relación con la periodontitis crónica.

Principales hallazgos. La presencia del alelo 2 en el polimorfismo del gen de la interleucina -1 beta y del genotipo positivo (2-2) es más frecuente en adultos peruanos con periodontitis crónica.

Implicancias. La identificación del alelo 2 en el polimorfismo de la IL-1B y el genotipo positivo (2-2) podría ser considerado en la prevención y tratamiento individualizado de la periodontitis crónica por parte de los odontólogos especialistas.

\section{EVALUACIÓN PERIODONTAL}

Odontólogos especialistas en periodoncia realizaron el diagnóstico del estado periodontal de los participantes. Los signos clínicos considerados fueron: formación de bolsas periodontales e inflamación gingival, sangrado al sondaje, movilidad dentaria aumentada, supuración y la medición de la profundidad del surco gingival. Según la extensión y severidad, los casos de periodontitis se clasificaron en: 1) periodontitis leve: cuando las pérdidas de inserción clínica son de uno a dos $\mathrm{mm}, 2$ ) periodontitis moderada: si las pérdidas de inserción se encuentran entre tres y cuatro $\mathrm{mm}$ y 3 ) periodontitis severa: ante pérdidas de inserciones clínicas mayores o iguales a cinco $\mathrm{mm}$.

\section{ANÁLISIS MOLECULAR}

EI ADN genómico fue aislado, según Balkwill $2000{ }^{(7)}$ y su cuantificación y calidad evaluada con el Nanodrop 2000. La genotipificación IL-1B C(+3953/4)T, se realizó amplificando una sección del exón 5 del gen de la IL-1B C(+3953/4)T, según la técnica de PCR-RFLP descrita por Balkwill $2000^{(7)}$. La misma que consiste en preparar una reacción de $50 \mu \mathrm{l}$, conteniendo $100 \mathrm{ng}$ de ADN, buffer Taq $10 \mathrm{X}\left(\mathrm{NH}_{4}\right) 2 \mathrm{SO}_{4}$, $0.8 \mathrm{mM}$ de dNTP mix, $25 \mathrm{mM} \mathrm{MgCl}, 1 \mu \mathrm{M}$ de cada uno de los primers (Foward: 5'-CTC AGG TGT CCT CGAAGAAAT CAAA-3' y Reverse: 5'-GCT TTT TTG CTG TGA GTC CCG3) y $2,5 \mathrm{u} / \mu \mathrm{L}$ de Taq Polimerasa Fermentas ${ }^{\circledR}$; el protocolo de amplificación fue: $95^{\circ} \mathrm{C}$ por dos min seguido de 35 ciclos (95 ${ }^{\circ} \mathrm{C}$ por un $\min , 67,5^{\circ} \mathrm{C}$ por un min, $72^{\circ} \mathrm{C}$ por un min) y $72^{\circ} \mathrm{C}$ por cinco min; los amplificados fueron visualizados en geles de agarosa Top Vision TMal 2,4 \% teñidos con Syber Green Invitrogen ${ }^{\circledR} 0,012 \%$, utilizando como step ladder Gene Ruler TM de 50-1000 pb Fermentas $®$, para luego ser digeridos a $65{ }^{\circ} \mathrm{C}$ durante cuatro horas con la enzima de restricción Taq I Fermentas $®$, considerando 15 unidades por $31,5 \mu \mathrm{l}$ de reacción; los productos (85 pb +97 pb +12 $\mathrm{pb}=$ alelo1 y $182 \mathrm{pb}+12 \mathrm{pb}=$ alelo 2) fueron visualizados en geles de poliacrilamida $8 \%$ teñidos con nitrato de plata usando como step ladder Gene Ruler TM de 50-1000 pb Fermentas $^{\circledR}$ (Figura 1). 


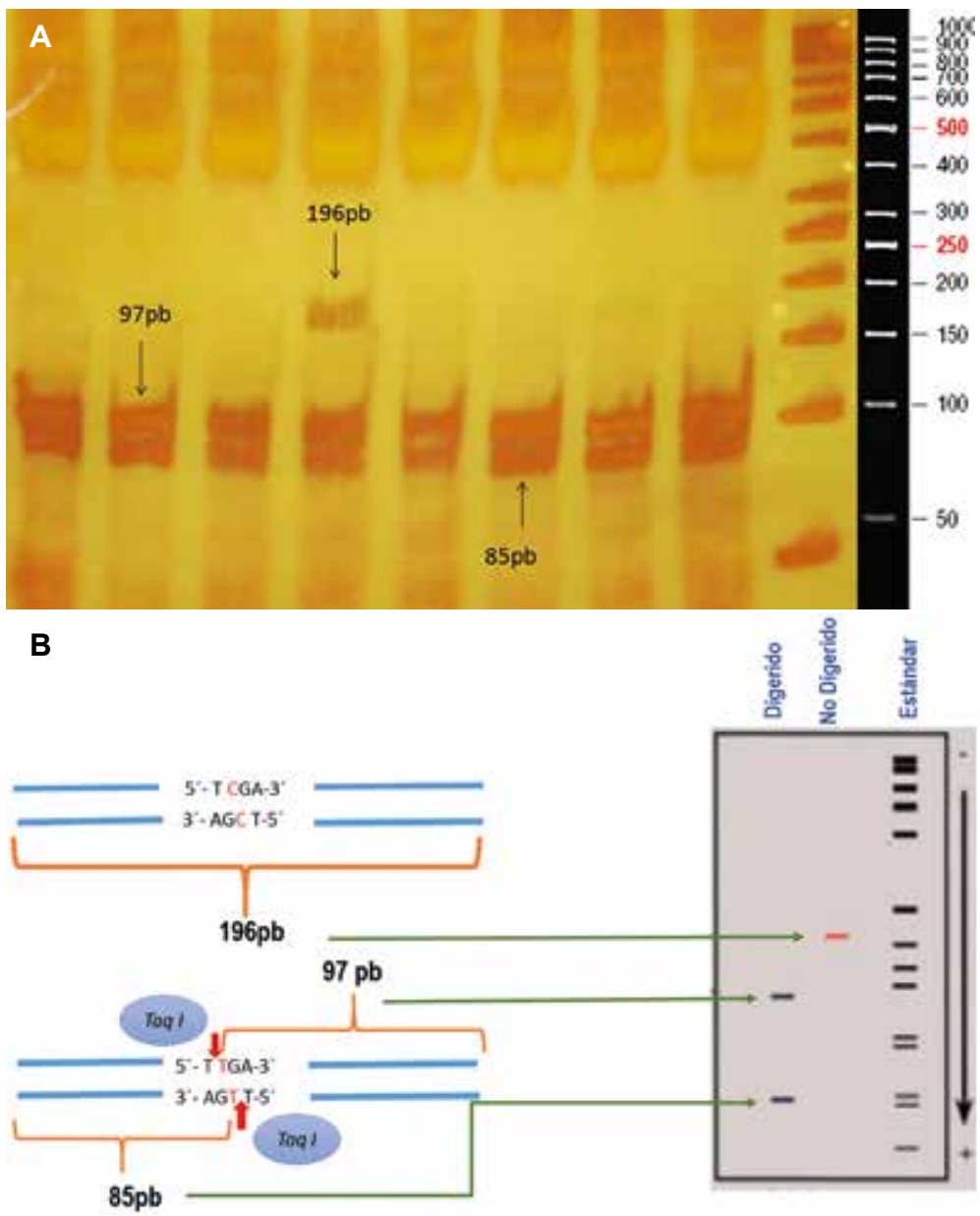

Figura 1. A. Fragmentos de restricción de IL-1B C(+3953/4)T de 196 pb, 97 pb y $85 \mathrm{pb}$ en gel de poliacrilamida al $8 \%$, tinción nitrato de plata y el patrón de bandas del ladder Gene Ruler TM de $50-1000$ pb, Fermentas ${ }^{\circledR}$. B. La enzima Taq I reconoce el sitio 5' - T CGA-3' 3'- AGC T-5', dentro del fragmento amplificado de la IL-1B; el genotipo homocigoto $\mathrm{CC}$ se determina al observar 2 bandas resultantes de la digestión (97bp + 85bp; alelo 1), el genotipo homocigoto TT, al observar una única banda de 196bp (alelo 2) y el genotipo heterocigoto CT se determina al observar 3 bandas (196bp, 97bp y 85bp).

\section{ANÁLISIS DE DATOS}

Se calculó el equilibrio de Hardy Weinberg (EHW) en la población control haciendo uso del test de Chi cuadrado, con un 0,05 de nivel de significación. Para determinar los factores asociados, se utilizó la regresión logística, empleando un nivel de significancia de 0,05 , y la razón de probabilidades (OR) con un $95 \%$ de intervalo de confianza (IC 95\%). El chi cuadrado de tendencia lineal se utilizó para determinar la asociación dosis-respuesta entre las variables. El análisis de datos se realizó empleando el paquete estadístico SPSS versión 17.0

\section{CONSIDERACIONES ÉTICAS}

Se obtuvo la aprobación del Comité de Bioética de la Facultad de Ciencias Biológicas de la Universidad Nacional Mayor de
San Marcos. Los participantes fueron informados; de los objetivos de la investigación, los procedimientos, riesgos y beneficios. Sólo los interesados en participar completaron una ficha de recolección de datos y el consentimiento informado.

\section{RESULTADOS}

\section{CARACTERISTIICAS DE LA POBLACIÓN DE ESTUDIO}

La población fue adulta joven, con más mujeres en los controles (58,3\%). Aproximadamente el $50 \%$ tiene estudios primarios y son nacidos en Lima. En relación a los servicios básicos, más del $90 \%$ cuentan con acceso a agua, desagüe y luz. El $50 \%$ tiene un frecuencia de lavado de los dientes de dos a más veces al día. Sin embargo, el uso de hilo dental y enjuagatorio bucal son poco frecuentes en esta población (Tabla 1). 
Tabla 1. Caracteristicas de la población estudiada

\begin{tabular}{|c|c|c|c|c|}
\hline \multirow{2}{*}{ Características } & \multicolumn{2}{|c|}{ Casos } & \multicolumn{2}{|c|}{ Controles } \\
\hline & $n=94$ & $\%$ & $n=96$ & $\%$ \\
\hline Edad (años) ${ }^{*}$ & \multicolumn{2}{|c|}{$46 \pm 13$} & \multicolumn{2}{|c|}{$36 \pm 14$} \\
\hline \multicolumn{5}{|l|}{ Sexo } \\
\hline Mujeres & 40 & 42,5 & 56 & 58,3 \\
\hline Hombres & 54 & 57,4 & 40 & 41,6 \\
\hline Nivel de estudio primaria & 49 & 52,1 & 43 & 44,7 \\
\hline Nacidos en Lima & 48 & 51,1 & 46 & 47,9 \\
\hline \multicolumn{5}{|l|}{ Ocupación } \\
\hline Ama de Casa & 27 & 28,7 & 35 & 36,4 \\
\hline Obrero & 37 & 39,3 & 22 & 22,9 \\
\hline Independiente & 12 & 12,7 & 14 & 14,6 \\
\hline Técnico & 10 & 10,6 & 12 & 12,5 \\
\hline Otros & 7 & 7,4 & 13 & 13,5 \\
\hline \multicolumn{5}{|l|}{ Servicios Básicos } \\
\hline Agua & 89 & 94,7 & 93 & 96,9 \\
\hline Desagüe & 93 & 98,9 & 92 & 96,9 \\
\hline Luz & 93 & 98,9 & 95 & 98,9 \\
\hline \multicolumn{5}{|l|}{ Higiene bucal } \\
\hline Cepillado de dientes 2 a más veces al día & 58 & 61,7 & 62 & 64,6 \\
\hline Usa hilo dental & 2 & 2,1 & 12 & 12,5 \\
\hline Usa enjuage bucal & 8 & 8,5 & 13 & 13,5 \\
\hline
\end{tabular}

*Edad media \pm desviación estándar

Se encontró que el polimorfismo IL-1B C(+3953/4)T se encuentra en $\mathrm{EHW}$ y la frecuencia genotípica predominante es el homocigoto 1-1, tanto en casos como en controles. Sin embargo, la frecuencia del genotipo 2-2 es mayor en los casos $(11,7 \%)$ (Tabla 2$)$.

\section{FACTORES ASOCIADOS CON PERIODONTITIS CRÓNICA}

Los factores asociados con la periodontitis crónica fueron ser mayor de 46 años (OR: 7,58, IC $95 \%$ : 3,32-17,30), tener grado de instrucción superior (OR: 0,43, IC 95 $\%$ : 0,27-0,98), asimismo la presencia del alelo 2 en el polimorfismo de la IL-1B y el genotipo positivo (2-2) estuvo asociado con la presencia de periodontitis crónica (OR: 2,06, IC 95 \%: 1,01-4,21).

\section{DISCUSIÓN}

La presencia del alelo 2 en el polimorfismo de la IL-1B y el genotipo positivo (2-2) le confiere al individuo mayor riesgo para el desarrollo de la periodontitis crónica ${ }^{(12)}$; por lo que se le considera un factor con implicancias a tener en cuenta en la medicina preventiva y tratamiento individualizado por parte de los odontólogos especialistas.

Estos resultados concuerdan con los hallazgos de varios autores ${ }^{(12-16)}$. La distribución de las frecuencias de los polimorfismos varían según el componente étnico de cada población, afectando la predisposición a la enfermedad periodontal y su prevalencia (12,13); en este caso, las distribución genotípica (2-2) para IL-1B C(+3953/4)T ( $11,7 \%$ en los casos y de $0 \%$ los controles), coinciden con las reportadas en Chile $(2,12 \%$ en los casos y $1,9 \%$ en los controles) ${ }^{(14) ;}$ y difieren de la población japonesa donde no se han encontrado homocigotos para el alelo $2{ }^{(13)}$. A nivel poblacional, las frecuencias del genotipo positivo (1-2/22 ) encontradas en este estudio $(27,7 \%$ en los casos y de $15,6 \%$ en los controles), son coincidentes con los reportes de la población chilena $(26,1 \%$ en los casos y de $9,9 \%$ en los controles ${ }^{(14)}$.

Tabla 2. Distribución del genotipo IL-1B C $(+3953 / 4) T$ en casos y controles en la población de estudio

\begin{tabular}{|c|c|c|c|c|}
\hline & \multicolumn{2}{|c|}{ Casos } & \multicolumn{2}{|c|}{ Controles } \\
\hline & $\mathrm{n}=94$ & $\%$ & $\mathrm{n}=96$ & $\%$ \\
\hline \multicolumn{5}{|c|}{ Genotipo IL-1B C(+3953/4)T } \\
\hline $1-1$ & 68 & 72,3 & 81 & 84,4 \\
\hline $1-2$ & 15 & 16,0 & 15 & 15,6 \\
\hline $2-2$ & 11 & 11,7 & 0 & 0 \\
\hline
\end{tabular}


Tabla 3. Asociación con la periodontitis crónica en la población de estudio

\begin{tabular}{|c|c|c|c|c|c|c|c|}
\hline \multirow{2}{*}{ Variable } & \multicolumn{2}{|c|}{ Casos } & \multicolumn{2}{|c|}{ Controles } & \multirow[b]{2}{*}{ OR } & \multirow[b]{2}{*}{ IC $95 \%$} & \multirow[b]{2}{*}{ Valor de $\mathrm{p}$} \\
\hline & $n=94$ & $\%$ & $n=96$ & $\%$ & & & \\
\hline \multicolumn{8}{|l|}{ Edad (años) ${ }^{*}$} \\
\hline $18-30$ & 11 & 21,6 & 40 & 78,4 & 1,00 & & 0,001 \\
\hline $31-45$ & 31 & 50,8 & 31 & 49,2 & 3,64 & $1,58-8,36$ & \\
\hline 46 a más & 50 & 67,6 & 24 & 32,4 & 7,58 & $3,32-17,30$ & \\
\hline \multicolumn{8}{|c|}{ Grado de instrucción ** } \\
\hline Primaria & 19 & 59,4 & 13 & 40,6 & 1,00 & & 0,038 \\
\hline Secundaria & 45 & 52,3 & 41 & 47,7 & 0,75 & $0,72-2,35$ & \\
\hline Superior & 23 & 38,3 & 37 & 61,7 & 0,43 & $0,27-0,98$ & \\
\hline \multicolumn{8}{|c|}{ Genotipo IL-1B C(+3953/4)T } \\
\hline$(1-1)$ & 68 & 72,3 & 81 & 84,4 & 1,00 & & 0,044 \\
\hline$(1-2) /(2-2)$ & 26 & 27,7 & 15 & 15,6 & 2,06 & $1,01-4,21$ & \\
\hline
\end{tabular}

* datos perdidos para dos participantes del grupo casos y un participante de grupo control

** datos perdidos para siete participantes en grupo de casos y cinco en grupo controles

Aunque algunos autores evidencian una posible asociación, en relación al número de alelo 2 presente; en nuestro estudio esta asociación no es clara. Posiblemente se deba que a pesar del rol funcional de los genes de la IL-1 en la transcripción de genes y producción de proteínas ${ }^{(7,15,16)}$, este se encuentra localizado en el exón 5 de la región codificante; y una substitución conservativa no podría afectar la transcripción del gen. No obstante, se han observado niveles elevados de IL-1 $\beta$ en el fluido crevicular gingival en pacientes portadores del genotipo positivo ${ }^{(17,18)}$. Así también, influiría la existencia de un efecto cooperativo

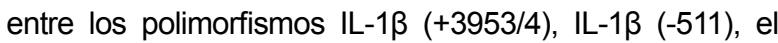
polimorfismo VNTR de la IL-1Ra ${ }^{(19)}$ y por la existencia de un desequilibrio de ligamento genético entre IL-1 $\beta(-511)$ y IL-1B (+3953/4) ${ }^{(20)}$.

Este estudio no ha considerado el polimorfismo de la región promotora de la IL-1B (-511) ni el polimorfismo VNTR de la IL-1Ra; al existir evidencia de la interrelación de estos polimorfismos con el polimorfismo IL-1B (+3953/4); por lo tanto, es posible que la presencia del alelo 2 en cualquiera de estos polimorfismos o en ambos favorezca la asociación del genotipo positivo de la IL-1B (+3953/4) con la enfermedad periodontal. Sin embargo, son necesarios mayores estudios que relacionen los genotipos de los tres polimorfismos (desequilibrio de ligamento genético entre los SNPs) y los niveles de IL-1B en el fluido cervicular gingival con la enfermedad periodontal.

En conclusión, los resultados del presente estudio apoyan la asociación entre la variación del polimorfismo genético de la IL-1B C(+3953/4)T y la periodontitis crónica; esta asociación coincide con las encontradas en poblaciones caucásicas, asiáticas y chilenas; no obstante, es necesario incluir en el análisis otros polimorfismos que pudieran estar relacionados con esta asociación.

Contribuciones de los autores: IFRM, JOG, MTV, PGS, AAA, AMYF, LAZC, ELP, EPG, han participado en la concepción del artículo, la recolección de datos, su redacción y aprobación de la versión final.

Fuente de financiamiento: El desarrollo de esta investigación contó con el apoyo económico del Fondo de Innovación de Ciencia y Tecnología FINCyT, Resolución Directoral N³0-2008-PCM/ FINCyT, contrato N001-FINCyT-PIBAP 2008 y se realizó en el Laboratorio II-5 de Genética Molecular del Centro de Investigación de Bioquímica y Nutrición de la Facultad de Medicina de la Universidad Nacional Mayor De San Marcos.

Conflictos de interes: Los autores declaran no tener concflictos de interés.

\section{REFERENCIAS BIBLIOGRÁFICAS}

1. Banjar W, Alshammari MH. Genetic factors in pathogenesis of chronic periodontitis. J Taibah Univ Med Sci. 2014;9(3):245-7. DOI: $10.1016 /$ j. jtumed.2014.04.003.

2. Otomo-Corgel J, Pucher JJ, Rethman MP, Reynolds $M$ a. State of the science: chronic periodontitis and systemic health. J Evid Based Dent Pract. Elsevier Inc.; 2012;12(3 Suppl):20-8. DOI: 10.1161/01. ATV.0000082462.26258.FE.
3. Bansal M, Khatri M, Taneja V. Potential role of periodontal infection in respiratory diseases - a review. J Med Life. 2013;6(3):244-8.

4. Klever da Silva M, Gonçalves de Carvalho AC, Pereira Alves EH, Pereira da Silva FR, Pessoa L dos $S$, Pereira Vasconcelos DF. Genetic Factors and the Risk of Periodontitis Development: Findings from a Systematic Review Composed of 13 Studies of Meta-Analysis with 71,531
Participants. Int J Dent. 2017;9. DOI: $10.1155 / 2017 / 1914073$

5. Wankhede A, Wankhede S, Wasu S. Role of genetic in periodontal disease. J Int Clin Dent Res Organ. 2017;9:53-8. DOI: 10.1161/01. ATV.0000082462.26258.FE.

6. Castrillón Rivera EL, Macín Cabrera AS, Palma Ramos A. Participación de la interleucina $1 \beta$ ( IL-1 $\beta$ ) en periodontitis. Rev Odontológica Mex. 2007;11(4):185-200. 
7. Balkwill F. Cytokine Molecular Biology A Practical Approach. Third edit. New York- U.S: Oxford University Press; 2000. 1-224 p.

8. Dosseva-Panova V, Mlachkova A, Popova C. Gene polymorphisms in periodontitis. Overview. Biotechnol Biotechnol Equip. Taylor \& Francis; 2015;29(5):834-9. DOI: 10.1080/13102818.2015.1056230.

9. Bushra K, Bhongade M, Pranav S, Priti C. Genetics: An Insight to Periodontal Disease. J Dent Med Sci. 2017;16(9):84-9. DOI: 10.9790/0853-1609098489.

10. Taboada Vega ME, Olivera García JE, Valderrama Herrera O, Cisneros Zárate L, Chuquihuaccha Granda V, Reyes Soto $S$, et al. Evaluación del Factor de Necrosis Tu- moral ( TNF-alfa) en pacientes perua- nos con procesos periodontales. Odontol Sanmarquina. 2007;10(1):28-30.

11. Ma L, Chu WM, Zhu J, Wu YN, Wang ZL. Interleukin-1 $\beta$ (3953/4) $\mathrm{C} \rightarrow \mathrm{T}$ polymorphism increases the risk of chronic periodontitis in Asians: Evidence from a meta-analysis of 20 case-control studies. Arch Med Sci. 2015;11(2):267-73. DOI: 10.5114/ aoms.2015.50961.

12. Deng JJ-S, Quin P, Li X-XX, Du Y-HY, Qin $\mathrm{P}, \mathrm{Li} \mathrm{X}-\mathrm{XX}$, et al. Association between interleukin-13 C (3953/4)T polymorphism and chronic periodontitis: evidence from a meta-analysis. Hum Immunol. 2013;74(3):371-8. DOI: 10.1016/j.humimm.2012.11.018.

13. Nikolopoulos GK, Dimou NL, Homodrakas SJ, Bagos PG. Cytokine gene polymorphisms in periodontal disease: a meta-analysis of 53 studies including 4178 cases and 4590 controls. J Clin Periodontol. 2008;35(9):754-67. DOI: 10.1111/j.1600-051X.2008.01298.x.

14. López NJ, Jara L, Valenzuela CY. Association of interleukin-1 polymorphisms with periodontal disease. J Periodontol. 2005;76(2):234-43. DOI: 10.1902/ jop.2005.76.2.234.

15. Baker PJ, Roopenian DC. Genetic susceptibility to chronic periodontal disease. Microbes Infect. 2002;4(11):1157-67. DOI: 10.1016/S1286-4579(02)01642-8.

16. Trevilatto PC, de Souza Pardo AP, Scarel-Caminaga RM, de Brito RB, Alvim-Pereira F, Alvim-Pereira CC, et al. Association of IL1 gene polymorphisms with chronic periodontitis in Brazilians. Arch Oral Biol. 2011;56(1):54-62. DOI: 10.1016/j.archoralbio.2010.09.004.

17. Valderrama G, Vijande F, Escribano J, Garrido-Pertierra A, Bascones A. La IL-1 y su eventual asociación con la enfermedad periodontal crónica .
Una revisión de la literatura ( I ). Av Periodon Implant. 2005;17(2):89-95.

18. Ferreira SB, Trombone APF, Repeke CE, Cardoso CR, Martins W, Santos CF, et al. An interleukin-1beta (IL-1beta) single-nucleotide polymorphism at position 3954 and red complex periodontopathogens independently and additively modulate the levels of IL-1beta in diseased periodontal tissues. Infect Immun. 2008;76(8):372534. DOI: 10.1128/IAI.00546-08.

19. Laurincová B. Interleukin-1 Family: From Genes To Human Disease. Acta Univ Palacki Olomuc. 2000;143:1930.

20. Bazrafshani M, Hajeer A, Ollier W, Thornhill M. IL-1B and IL-6 gene polymorphisms encode significant risk for the development of recurrent aphthous stomatitis (RAS). Genes Immun. 2002;3(5):302-5. DOI: $10.1038 / \mathrm{sj}$. gene.6363882.

Correspondencia: Ivonne Fanny Reyes Mandujano Dirección: Jr. Los Sociólogos Mz A Lt 1-A Coop. Huancaray - Canto Rey - San Juan de Lurigancho

Telefono: 969773204

Correo electrónico:ivonnefanny@gmail.com

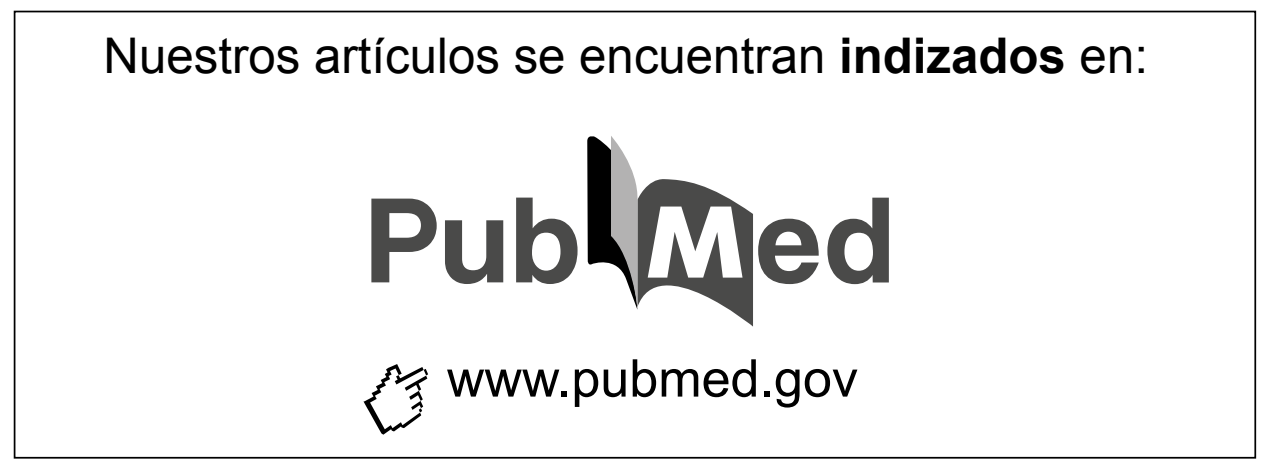

